\title{
Philosophy for Communites al Liceo "Govone" di Mondovì (Italy)
}

\author{
Silvia Bevilacqua, Pierpaolo Casarin
}

(Propositi di filosofia e Insieme di pratiche filosoficamente autonome, bevilacqua77@yahoo.it, pier@pierpaolocasarin.it)

Qui di seguito troverete alcune riflessioni scaturite a partire da un progetto nell'ambito della IX edizione (2016) della Summer School: Futuri, immaginare il mondo di domani organizzato dall'Associazione CeSPeC. L'idea di fondo dell'iniziativa è stata quella di proporre una molteplicità di incontri, approfondimenti e dibattiti intorno alla riflessione sul tempo presente con un'attenzione particolare a concetti come: creatività, legami, affettività, diversità, giustizia, educazione intesi come lessico per pensare il futuro e orientare pratiche di trasformazione sociale culturale e formativa. In quest'ottica è stato proposto un progetto di philosophy for communities alle scuole, ed in particolare alle classi IVA Scientifico e IVB-VB di Scienze umane del Liceo Vasco-Beccaria-Govone di Mondovì l'intervento ha preso avvio il 17 settembre 2017 per proseguire durante l'anno scolastico. Il percorso ha trovato articolazione in una serie di attività di pratica di filosofia/philosophy for communities come esperienze di pensiero riflessivocritico fra i ragazzi e le ragazze che frequentano il Liceo "Vasco-BeccariaGovone". In questa prospettiva la filosofia è intesa come messa in gioco dei soggetti, creazione di concetti, esercizio di ricerca e argomentazione in particolare attraverso l'attività del domandare. Viene assegnata, in questa dimensione, precedenza al filosofare, al pensare non al filosofato e al pensato. Una filosofia che, così facendo, mostri la possibilità di ritornare essa stessa $a$ scuola per rimettersi profondamente in gioco, per ri-articolare le sue modalità, per rivisitare la geografia dei suoi saperi. Una pratica che ispirandosi alla philosophy for children intenda la scuola come una comunità di ricerca, un luogo di transizione, in cui sviluppare forme creative di cooperazione, per costruire disposizioni e sensibilità che possano affrontare le complessità sociali e culturali della nostra epoca. Quella della pratica della filosofia, intesa non come luogo d'eccezione del pensiero di alcuni, ma come luogo democratico di articolazione del pensiero, del dialogo e della riflessione critica, ci è parsa una possibilità interessante e un terreno proficuo di sviluppo. In questo senso la filosofia diviene spazio e luogo non solo del sapere, ma soprattutto del pensare, dell'incontro con il proprio e altrui pensiero; un'uscita dai confini, un'apertura ad essere attività che agisce concettualmente in relazione a ciò di cui si fa esperienza. Un progetto che grazie all'accoglienza 
e sensibilità filosofica della Prof.ssa Angela Cucchi e del Prof. Riccardo Lubatti ha avuto un esito proficuo e interessante con una partecipazione significativa e coinvolta dei ragazzi e delle ragazze. A partire dalle attività di dialogo e da alcune riflessioni scritte che alla fine del progetto ci sono state restituite e abbiamo pensato di entrare in relazione in queste righe. Una sorta di dialogo aperto per pensare ancora insieme. In corsivo le considerazioni che i ragazzi e le ragazze hanno maturato in relazione all'esperienza e di seguito alcune nostre "reazioni" filosofiche e riflessive sulla pratica e non solo.

Al termine del progetto abbiamo chiesto ai ragazzi e alle ragazze di esprimersi, attraverso un esercizio di scrittura, intorno ad alcuni aspetti della pratica. Le profonde sollecitazioni emerse ci hanno spinto a mettere in evidenza alcune questioni che riportiamo qui di seguito. Troverete in neretto le riflessioni dei ragazzi e delle ragazze e di seguito le nostre "reazioni" filosofiche e metariflessive.

\section{I pensieri pensati insieme sono migliori dei pensieri pensati da soli}

Questa affermazione è una delle considerazioni meno comuni quando si parla di filosofia e proprio per questo ci sembra particolarmente interessante. La tradizione vuole che un filosofo o filosofa pensino per lo più fra sé e sé, in solitudine e con rigore le questioni che nel corso della storia del pensiero occidentale si sono susseguite. Tuttavia è lecito pensare che nessuno possa essere veramente da solo a pensare, poiché il proprio pensiero è in continuo attrito e reagisce a concetti dentro e fuori di noi. Hannah Arendt ci ha mostrato, che già nel conosci te stesso delfico, c'è in gioco conoscenza della propria esistenza e che nel so di non sapere socratico si annida la necessità di conoscere e avvicinarsi all'altro e alle sue opinioni. In questo Arendt mette in evidenza un aspetto importante:

nell'esperienza del parlare con sé stessi si diviene capaci di essere amici, di acquistare un altro da sé: la facoltà del discorso e il fatto della pluralità umana, allora si corrispondono reciprocamente, non solo nel senso che impieghiamo delle parole per comunicare in un mondo in cui viviamo insieme ad altri, ma anche nel senso, ancor più rilevante, che parlando con noi stessi viviamo insieme $a$ noi stessi. [...] Essendo uno io non mi contraddirò al tempo stesso potrò contraddirmi, poiché nel pensiero io sono-due-in-uno e quindi non vivo soltanto con gli altri, per i quali sono uno ma anche con me stesso. La paura della contraddizione è paura della scissione, del non poter restare uno; questo spiega perché il principio di non-contraddizione sia divenuto la regola fondamentale del Pensiero (Arendt 2015, 41).

Siamo d'accordo con Arendt, ma l'attività di dialogo contempla un pensiero pensato insieme che rende più complessa e vitale questa condizione di per sé 
molteplice, che si sperimenta interiormente. Lo spazio del confronto ci mette in gioco come soggetti esposti a regole impreviste in cui è necessaria una concentrazione, un ascolto e una disposizione intensa e talvolta molto faticosa. Un pensare differente fra le differenze in cui la pluralità umana e la complessità che ne deriva non subiscono una riduzione, ma una moltiplicazione; a scapito, talvolta, di una comunicazione efficace, ma comunque a favore di un pensare senza corrimano che senza gli abituali sostegni ci spinge, appunto, la nostra individualità e le nostre credenze. Perciò anche se il rapporto con il testo filosofico o con il nostro pensiero ci mette nella condizione di sperimentare la pluralità, il dialogo fra persone conduce ad altre virate e condizioni di differenza date dalla contingenza di un altro presente. In questo senso nell'espressione «i pensieri pensati insieme sono migliori dei pensieri pensati da soli» risiede non tanto una differenza di qualità quanto una differenza vitale: quel bisogno di sentirsi coinvolti, insieme ad altri/e, in un nuovo rapporto con la filosofia, con le sue domande e con le questioni che nel mondo intercettiamo, un impegno politico e pratico.

La filosofia, in questa luce, accentua maggiormente il valore e la possibilità di un pensare insieme collettivo che probabilmente tralascia, solo temporaneamente, un po' di rigore, una certa spinta definitoria e la scrittura. Diciamo temporaneamente perché riteniamo che questa esperienza non annulli la possibilità di un rapporto classico con il testo filosofico, ma lo trasformi, lo alimenti allentando però quella posizione individualista e solitaria che abbiamo del filosofo/a a favore dell'idea di una comunità di ricerca.

Ma quando ha inizio questo pensare insieme? Quando quel pensiero collettivo a cui fanno accenno alcune delle riflessioni dei ragazzi/e trova spazio nell'ambito del tempo della scuola e di quello sociale? Quando le proprie idee differenti hanno la possibilità di trovare luogo e di essere messe in relazione a quelle di altri/e? Possiamo ritenere che le pratiche di filosofia e la philosophy for children/communities siano questa potenzialità di apertura? In questa prospettiva ci sembra importante raccogliere l'invito di Michel Foucault quando propone un'idea di filosofia come moto attraverso cui ci liberiamo da ciò che si propone vero a tutti i costi; in questo senso il filosofare rende possibile il «processo critico del pensiero su se stesso [...] che invece di legittimare ciò che già si conosce, cerca di sapere in che modo e fino a dove sarebbe possibile pensare in modo divergente» (Foucault 1986, 12), una predisposizione al molteplice, una ricerca permanente, sincera, per essere differenti da ciò che siamo e per costituirci in ciò che ancora non siamo. Un'esperienza che ogni soggetto, a prescindere dalla sua età, può provare ad inaugurare. Così, sempre in questa prospettiva, Foucault rispondeva ad alcune sollecitazioni di Duccio Trombadori: «Io scrivo proprio perché non so ancora cosa pensare di un argomento che attira il mio interesse. Facendolo, il libro mi trasforma, muta ciò che penso; di conseguenza, ogni nuovo lavoro cambia 
profondamente i termini di pensiero cui ero giunto con quello precedente» (Trombadori 1999, 30).

\section{Cogliere significati nascosti, rielaborare il punto di vista personale}

La visione degli altri, il punto da cui ognuno/a si posiziona è differente e spesso in contrasto con la nostra tonalità di sguardo. Non solo, ma come scrive Wittgenstein in uno dei suoi passaggi dei Pensieri Diversi: «Quel che voglio esprimere lo esprimo sempre e soltanto a metà! Anzi, neppure tanto, forse riesco a esprimere solo la decima parte. Questo vorrà pur dire qualcosa. Il mio scrivere è spesso solo un "balbettare"» (Wittgenstein 1980, 47). Non solo lo scrivere, ma anche nell'espressione a voce si incorre in questa complessità. La ricerca nella pratica filosofica, la disposizione a ricercare, può rappresentare un modo attraverso cui questo "contrasto e differenza, questa fatica dell'esprimere ciò che si pensa e del comprendere di conseguenza ciò che l'altro esprime» prende vitalità: «Poiché il mondo sconfina su di noi, la parola può sconfinare su di esso esprimendolo, e l'azione trasformandolo. Si filosofa perché si è esposti al mondo e si ha la responsabilità di nominare ciò che deve essere detto o fatto» (Lyotard 2015, xiv).

Si tratta di una questione di desiderio, una direzione che conduce verso l'altro, una condizione di incompletezza che ci permette di ricercare ancora significati, un continuo lavorio di termini, parole, esperienze che spinge oltre il sapere a favore di un ritornare della filosofia ad essere una "faccenda umana" che, parafrasando Karl Jaspers, non intende confermare dottrine, ma mantenere aperte domande. Si osserva così nel pensare insieme la messa in atto di un ulteriore spazio di conoscenza che non riposa su risposte certe e definitive da acquisire, ma si addentra nella ricerca comune e permanente. Questo cogliere i significati nascosti ci riporta ad un sapere transitorio che ricerca ancora il desiderio di pensare e di pensiero. La vita ha bisogno di pensiero e viceversa, è quasi inumano ritenere che la filosofia possa essere considerata solo nella sua forma tradizionalmente intesa, quella che ha prevalso storicamente sulle altre ovvero come sviluppo di sistematicità; è importante ritornare a pensare che vi sia una molteplicità di forme della filosofia che sappiano trovare la propria generatività nell'urto con la vita.

La vita ha bisogno di pensiero e viceversa, è quasi inumano ritenere che la filosofia possa essere considerata solo nella sua forma tradizionalmente intesa, quella che ha prevalso storicamente sulle altre ovvero come sviluppo di sistematicità? Si può pensare che vi sia una molteplicità di forme della filosofia che sappiano trovare la propria generatività nell'urto con la vita?

Come ricorda Zambrano: «la vita ha bisogno del pensiero, ma ne ha bisogno perché non può preservare lo stato in cui spontaneamente si produce. Non basta infatti nascere una volta e muoversi in un mondo di strumenti utili. La 
vita umana chiede sempre di essere trasformata, di modificarsi continuamente a contatto con certe verità» (Zambrano 1996, 58). Questa apertura allo spazio filosofico del pensare insieme fuori da una logica applicativa e ripetitiva è un aspetto fondamentale. In questa luce siamo nella condizione proficua di riflettere intorno a ciò che è stato trasmesso e, contemporaneamente, di accogliere le molteplici traiettorie che si prospettano. Non si tratta di lanciarsi acriticamente verso un nuovo modo, indefinito o spontaneistico, di intendere la pratica filosofica, ma nemmeno di accettare un unico rapporto, talvolta soffocante, con un tradizione intesa in termini immodificabili. In questione la nostra capacità di trasformare, il nostro desiderio di uscire dallo stereotipo e dalla cristallizzazione delle retoriche, talora logore, della procedura ${ }^{1}$.

\section{Domande da fare, non da esaurire e a cui non si sono suggerite risposte}

Uno degli aspetti centrali della philosophy for children/communities è l'attività del domandare. Un domandare che porta con sé un'attitudine infantile che come un invito richiama:

ad una certa spontaneità, al desiderio, al bisogno che l'interrogazione porta con sé; non lasciare che una questione, un pensiero o una parola possano, troppo facilmente, esaurirsi in modo definitivo. Non solo il concetto infantile porta con sé, dal nostro punto di vista, una certa tensione alla ricerca, ovvero una conoscenza non sottomessa l'accumulo sterile del sapere. Il domandare si esprime con questa vitalità se lo si prende in considerazione come ad una possibilità permanete, un'apertura all'indagine continua a costo di rimescolare le carte e rimettere in discussione tutto quanto (Bevilacqua \& Casarin 2016, 55).

Il domandare è il tempo e lo spazio per pensare non per giungere a risposte necessarie o veritative, è una disposizione alla relazione con l'altro, ad una tensione verso ciò che sembra mancare e che proprio perché vuoto invita a immaginare, creare, inventare ragionare ovvero ad entrare in relazione alle nostre potenzialità di pensiero: «Tra la domanda e la risposta deve esistere un vuoto, una mediazione, un soffermarsi della mente» (Zambrano 2008, 37). Fare una domanda sembra un'operazione semplice, ma non è così. Fare una domanda implica attenzione, ascolto, disposizione al dubbio, sospensione di una certa retorica e presunzione veritativa che attribuiamo alle nostre riflessioni.

Fare una domanda implica altresì un rapporto con chi ho di fronte e che insieme a noi s'impegna e si fa coinvolgere ad esplorare le questioni che si pongono. Si tratta di sperimentare un legame di reciprocità che nel dialogo

\footnotetext{
${ }^{1}$ In merito ad una riflessione più dettagliata del rapporto fra metodologia, dispositivo di potere e philosophy for children si veda il saggio Bevilacqua \& Casarin $(2016,55)$.
} 
invita, non tanto a posizionarsi in virtù dell'argomento più forte o con più ragioni, ma piuttosto in favore della differenza di opinioni, di punti di vista nell'idea di una provocazione generativa e propositiva per far germogliare idee e concetti vivi. In merito a questo è molto interessante una delle considerazioni che uno dei ragazzi ha messo in evidenza: questa esperienza di pratica di filosofia ha fatto sì che ci si avvicinasse alla compagna di banco seduta due file avanti e che per lungo tempo ho discriminato per via di un pregiudizio. La conoscenza dell'altro/a passa dall'ascolto del pensiero, delle idee e delle riflessioni che si esprimono in un clima di fiducia e sospensione del giudizio. Siamo innanzi alla costruzione di una comunità di ricerca fondata sulla condivisione, la creazione e ricreazione concettuale.

\section{La pratica di filosofia è utile alla formazione umana, è un affrontare il fondo melmoso dei nostri pensiero, una libera ricerca, un'esperienza di pensiero collettivo}

Non basta forse dire che fare filosofia sia un modo per educare alla riflessione, al dialogo o al pensiero. Certo questi sono aspetti importanti, ma non l'unico senso che può emergere da queste attività ed esperienze. C'è qualcosa che procede oltre, che va a sollecitare l'idea che in esse si respiri una boccata d'aria, che si faccia i conti con la condizione umana del vivere insieme, la medesima che Arendt ci ha suggerito di pensare:

La pluralità umana, condizione fondamentale sia del discorso sia dell'azione, ha il duplice carattere dell'eguaglianza e della distinzione. Se gli uomini non fossero uguali non potrebbero né comprendere fra loro, né comprendere i propri predecessori, né fare progetti per il futuro e prevedere la necessità dei loro successori (Arendt 1994, 127).

Una formazione che non fa solo i conti con capacità e competenze, ma con la soggettività di ognuno/a in uno spazio comune. Una soggettività che spesso in modo melmoso cerca di dipanarsi nei propri silenzi personali e ritirati e che non così spesso trova spazio per esprimersi, mettersi in discussione e confrontarsi. Una libera ricerca dunque che è libera perché non deve sottostare a un "sapere già pensato" già predisposto o a una erudizione cristallizzata, ma si apre alla ricerca perché non soddisfatta dalle posizioni che dentro di noi abbiamo già raggiunto. Un tempo ed una pausa da una certa abitudine scolastica o di vita che non ha tempo per fermarsi a pensare, ma che perlopiù si consuma senza il tempo per la riflessione. Una comunità di ricerca o un'esperienza di pensiero collettivo che nella filosofia ritrova la possibilità di farsi evento qui inteso in senso deleuziano:

la filosofia di Deleuze è sempre una filosofia dell'evento, impegnata a ritrovare e celebrare dietro la durezza solidificata 
dei fatti e delle cose (ma anche degli io, delle istituzioni, delle teroie filosofiche) l'esperienza pura, il Reale, il divenire-nomi diversi che cercano tutti di nominare un essere primo che è potenza, energheia, divenire, processi che accade prima della costituzione di qualsiasi soggetti (Peverelli 2017, 11).

Un'eventualità appunto che possiamo raccogliere, sostenere e immaginare di moltiplicare nelle molte situazioni comunitarie in cui ci ritroviamo. Una filosofia fuori classe che ne dilata i confini, che non contiene e lascia che si generi una forma spontanea, inaspettata non prevista. La ricerca filosofica come spazio assume una connotazione politica delle relazioni e del sapere come impegno a mantenere nella nostra ricerca filosofica uno stile plurale, collettivo, attento a individuare e, se possibile, a disattendere dinamiche di dominio. Ciò significa esporsi a un'idea di pensiero senza balaustre, contro ogni tirannia del vero a tutti i costi, a favore dell'uguaglianza e della pluralità sembrano essere un punto essenziale per la philosophy for children/communities. A sostenere un'idea affine è Rossella Fabbrichesi che in un interessante saggio dal titolo: Cosa si fa quando si fa filosofia?, mette in luce il fatto che nel tempo immemorabile del fare filosofia, si muove un tempo di scambi comuni che è:

intrinsecamente politico, come si può ben capire. Mira a costruire una comunità di ricerca, fondata sulla condivisione degli interessi e sull'invenzione di nuove pratiche collettive: per non dimenticare mai come la filosofia sia l'unica difesa dalle mitologie con cui il tempo presente possa incantarci (Fabbrichesi 2017, xiv).

Un'apertura verso una prospettiva politica all'insegna della partecipazione attiva, del principio della reciprocità egualitaria, dell'impegno collaborativo, della conoscenza come azione condivisa e responsabile. La comunità di ricerca, riprendendo il pensiero di Dewey, evidenzia la stretta connessione tra dimensione etica, politica e conoscitiva. Per Dewey il presupposto principale della valutazione d'ogni azione in vista di un obiettivo è rappresentato dal riconoscimento dello stretto legame esistente fra mezzi e fini; e con un certo accento libertario potremmo mettere in evidenza la precedenza attribuita, nel nostro modo di declinare la comunità di ricerca, alla cura dei modi piuttosto che al raggiungimento degli obiettivi.

\section{La pluralità di testi e di temi sono un valore aggiunto}

A partire da questa sollecitazione che emerge dai ragazzi s'intende approfondire ciò che nell'accezione lipmaniana è il testo pre-testo che invita a pensare. Lipman ha creato una serie di racconti, delle fiction, che hanno principalmente due caratteristiche: primo sono testi di transito utili a 
stimolare domande e questioni aperte e sono caratterizzati da assenza di morale e pluralità di tematiche filosofiche, secondo sono costruiti come testi "modellanti" ovvero scritti che nella loro funzione stimolano a "rispecchiarsi e immedesimarsi" nelle vicende dei personaggi che vivono esperienze con caratteristiche vicine alla pratica che stiamo svolgendo in quel momento (dialogano, fanno domande, ragionano, argomentano ecc.). Dunque se da un lato questi testi sospendono una certa forza veritativa e di conseguenza attenuano il "linguaggio-codice" tipico della filosofia, dall'altro mantengono le tematiche filosofiche "tradotte" e inserite fra le righe. In questo modo chi legge il pre-testo lipmaniano entrerà in relazione con un sottile e indiretto invito a porre domande intorno ad alcune questioni. Il secondo aspetto, che potremmo ritenere un dispositivo, sostiene la struttura educativa che questi testi intendono avere al fine di facilitare, proprio attraverso una sorta di "esempio, modello", la modalità attraverso cui la comunità di ricerca e i soggetti in gioco dovrebbero muoversi. Questa intenzione non è evidente ai partecipanti, non si dice cosa e come si deve essere, ma ogni personaggio e situazione ricalca e riprende le caratteristiche fondamentale della comunità di ricerca (l'insegnante come facilitatore, il domandare, la riflessione lo scambio democratico fra i soggetti). Ciò rappresenta un punto fondamentale nell'idea educativa lipmaniana. Questo piano fra realtà (i personaggi sono molto simili a chi sta leggendo ovvero i testi tengono in considerazione il contesto, l'età, ecc. di chi li leggerà) e la finzione articolano un dispositivo che non è solo un pretesto per pensare, ma per "formarsi secondo il modello" come comunità di ricerca.

È chiaro che quando s'intende allargare leggermente i confini di questo spazio tale genere di testo diviene direttivo ed espressione di un modello e di un'idea di pratica di filosofia molto definita. Proprio in virtù di un'intenzione dinamica della costruzione della comunità di ricerca e dello stile del facilitatore/trice e dell'andamento del dialogo si è deciso di prendere in considerazione una pluralità di testi attingendo dal panorama filosofico, letterario e lipmaniano al fine di sospendere una certa prevalenza del dispositivo educativo che il testo lipmaniano produce nel facilitatora/trice e nei/nelle partecipanti. A ispirarci c'è un'idea di fondo: «aprire le porte della classe, rivisitare "il cerchio" della philosophy for children, per mettere in questione possibili rigidità metodologiche, accogliere eventuali imprevisti per favorire ulteriori spunti di ricerca, al fine di immaginare inedite traiettorie di pratica» (Bevilacqua \& Casarin 2016, 26).

L'auspicio è quello di realizzare una filosofia a scuola che non si mostri certo solo come dispositivo o ripetizione di insistite e invariate metodologie, ma che intenda essere essa stessa sperimentazione, rivisitazione della complessa trama dei suoi saperi; una disponibilità ad aprirsi a nuove forme e smascherare antiche lacune. 
Questa scelta è stata motivata anche da un altro aspetto. Se il testo lipmaniano ha la caratteristica positiva di alleggerire un certo stile filosofico anche attenuando l'affermazione di verità o morali definitive, semplificando la messa in gioco delle questioni filosofiche, sospende così anche quello straordinario attrito di complessità argomentativa, di concetto e linguistica che il testo filosofico possiede. Una complessità argomentativa e tematica che non è da assumere come sapere, da studiare e ripetere, ma utile ad una frizione concettuale in cui il soggetto dovrebbe mettersi in gioco senza preoccuparsi dell'autorità del testo stesso o dell'autore/trice in questione.

Inoltre con un testo non lipmaniano non ci troviamo di fronte a un "modello di dialogo e di ragionamento", ma entriamo in relazione al molteplici stili e modi che ogni testo, pensatore, pensatrice offrono. Per far sì che questo rapporto con il testo non si trasformi in uno esercizio di erudizione dobbiamo, a nostro avviso, tenere in considerazione due aspetti importanti della proposta lipmaniana: che ogni cosa espressa non venga ritenuta del tutto giusta o del tutto sbagliata (dunque non si parla per dire cosa ha veramente detto l'autore o l'autrice, si sospende dunque un certo sapere-potere lasciando spazio ad altre domande, interpretazioni e pensieri) e che si proceda verso la valorizzazione di un ascolto rivolto a ciò che l'altro o l'altra dicono sulla base degli argomenti, delle riflessioni e degli eventuali esempi che riportano in relazione al pensiero che esprimeno al fine di generare quella particolare condizione filosofica che Deleuze e Guattari indicano come creazione concettuale. Per questi due pensatori la filosofia stessa è l'arte di formare concetti ciò implica che vi sia un'impegno attivo e non passivo nell'attività di pensiero e che la filosofia così intesa non sia solo il «ricevere un pensato per chiarirlo, spiegarlo e purificarlo», ma possa essere un'attività fabbrile, artigiana ovvero imparare a pensare, conoscere far si che l'ovvio non ci attraversi come ovvio (Deleuze-Guattari 1991).

In questo modo la nostra idea di comunità di ricerca non si costruisce sulla base di un processo educativo o filosofico procedurale, ma si mette in atto in relazione a ciò che tendiamo e desideriamo mettere in gioco; le domande, il dialogo, il rapporto fra le persone che nascono sono meno immediate, ma esito di un'attività intensa, coinvolgente e non solo automatica, retorica, metodologica. In questa prospettiva intendiamo parlare di post philosophy for children, un movimento, un tragitto che intende valorizzare alcuni aspetti della philosophy for children, ma che desidera, al tempo stesso, articolare altre occasioni di comprensione, riflessione e pratica in cui la stessa idea di filosofia potrebbe nuovamente "mettere al mondo" la domanda: che cos'è la filosofia? E ancora: Che cosa intendiamo per filosofia pratica e per filosofia teoretica? In fondo fu proprio Matthew Lipman, nella prefazione al manuale di Elfie, uno degli scritti del suo curricolo, ad indicare questa linea, invitando i genitori e gli insegnanti a incontrare la philosophy for children immaginandosi compagni e compagne di viaggio in cui immergersi e da cui farsi sbalordire e sorprendere. 


\section{Literature}

Arendt, H. 1994. Vita Activa. Milano: Bompiani.

Arendt, H. 2015. Socrate. Milano: Raffaello Cortina.

Bevilacqua, S. \& Casarin, P. (a cura di). 2016. Philosophy for children in gioco.

Milano-Udine: Mimesis.

Deleuze, G. \& Guattari, F. 1991. Che cos'è la filosofia? Torino: Einaudi.

Fabbrichesi, R. 2017. Cosa si fa quando si fa filosofia? Milano: Raffaello Cortina.

Foucault, M. 1986. Storia della sessualità, Vol. II. Milano: Feltrinelli.

Lyotard, J.-F. 2015. Perché la filosofia è necessaria? Milano: Raffaello Cortina.

Peverelli, R. 2017. Introduzione. In G. Deleuze, La fine degli intellettuali. Conversazioni con Gilles Deleuze. Milano: Medusa.

Trombadori, D. 1999. Colloqui con Foucault. Roma: Castelvecchi.

Wittgenstein, L. 1980. Pensieri diversi. Milano: Adelphi.

Zambrano, M. 1996. Verso un sapere dell'anima. Milano: Raffaello Cortina.

Zambrano, M. 2008. Per l'amore e per la libertà, scritti sulla filosofia e sull'educazione. Genova-Milano: Marietti. 
Silvia Bevilacqua (Genova), Pierpaolo Casarin (Milano)

(Propositi di filosofia e Insieme di pratiche filosoficamente autonome, bevilacqua77@yahoo.it, pier@pierpaolocasarin.it)

\title{
Philosophy for Communities al Liceo "Govone" di Mondovì (Italy)
}

\begin{abstract}
Italian). In questo contributo si riportano alcune riflessioni scaturite a partire dal progetto di philosophy for communities nelle scuole del Liceo "Vasco-Beccaria-Govone" di Mondovl (Italia) nell'ambito della IX edizione (2016) della Summer School Futuri, immaginare il mondo di domani organizzato dall'Associazione CeSPeC. La filosofia e intesa come prassi, esperienza di messa in gioco dei soggetti, creazione di concetti, esercizio di ricerca e argomentazione in particolare attraverso l'attivita del domandare. A partire dalle attivita di dialogo e da alcune riflessioni scritte dai ragazzi e dalle ragazze e scaturito una sorta di dialogo aperto per pensare ancora insieme. In questa riflessione si propone l'idea della post philosophy for children, un movimento, un tragitto che intende valorizzare alcuni aspetti della philosophy for children, ma desidera, al tempo stesso, articolare altre occasioni di comprensione, riflessione e pratica in cui la stessa idea di filosofia potrebbe mettere al mondo nuovamente le domanda: che cos'e la filosofia?
\end{abstract}

\begin{abstract}
English). In this contribution we shall focus on the project of philosophy for communities carried out at the Liceo "Vasco-BeccariaGovone" (Mondovı, Italy) within the IX edition (2016) of the CeSPeC Summer School on Futures, imagining the world of tomorrow. Philosophy is understood as a practice, an experience, a creation of concepts, an inquiry, as an exercise of argumentation and research. Thanks to this view, a dialogue has opened up with the pupils of this school. In this contribution we present the perspective of a post-philosophy for children and we understand it as an opportunity for philosophy in itself.
\end{abstract}

Keywords: post philosophy for children/communities, practices, thinking, inquiry, in-between

Ethics in Progress (ISSN 2084-9257). Vol. 8 (2017). No. 1, Art. \#16, pp. 252-262.

Creative Commons BY-SA 3.0

Doi: 10.14746/eip.2017.1.16 